\title{
New Onset Viral COVID19 Infection in Two End Stage Renal Disease Patients on Extracorporeal Hemodialysis Treatment
}

\author{
Ferrara $G^{1^{*}}(\mathbb{D})$, Guida $C C^{1}$, De Cosmo $S^{2}$ and Aucella $F^{1}$ \\ ${ }^{1}$ Department of Nephrology and Dialysis, Fondazione IRCCS "Casa Sollievo della Sofferenza", San Giovanni Rotondo (FG), \\ Italy \\ ${ }^{2}$ Department of Medical Sciences, IRCCS "Casa Sollievo della Sofferenza", Scientific Institute for Research and Health \\ Care, San Giovanni Rotondo, Italy
}

\begin{abstract}
From December 2019 an outbreak of novel coronavirus disease has represented the major global human threat spreading rapidly worldwide and turning into a pandemic [1]. The World Health Organization (WHO) named this new virus Severe Acute Respiratory Syndrome Coronavirus 2 (Sars-CoV-2) and the related disease was defined as coronavirus disease 2019 (COVID-19) [2]. COVID-19 is a highly infectious, rapidly spreading viral disease with an alarming fatality rate up to 5\% [3]. A matter of great interest is the wide spectrum of COVID-19 symptomatology and disease manifestation, ranging from completely asymptomatic carriers to critical respiratory failure, multiorgan dysfunction, and death [4]. The most common symptoms are like those of the common seasonal flu and cold and the WHO has considered dry cough and fever as the common symptoms of COVID-19 [5]. The risk factors for severe presentations are concentrated in patients with comorbidities such as advanced age and cardiovascular and chronic degenerative diseases particularly patients with end-stage renal disease (ESRD) who are dialysis dependent. A better characterization of COVID-19 infection in critically ill patients is important to guide decision making regarding critical care capacity and allocation of resources [4]. We report two cases of dialysis patients on a weekly three days phasing at their own centers who were admitted in hospital for different pathologies and then developed COVID-19 infection.
\end{abstract}

\section{Keywords}

Chronic renal failure, Chronic hemodialysis, COVID 19 over infection

\section{Case Report}

The first patient is a 62-years-old Caucasian male, affected by adult polycystic kidney disease in extracorporeal hemodialysis through an arterio-venous native fistula. The subject had presented fever for about a month and a half before being admitted. The diagnosis was cist's infection and abscessualization of one of them (polycystic kidney cists') which required the surgical removal of both kidneys. The diagnosis was achieved by performing abdominal ultrasound eco-scan and Computed Total body tomography, followed by marked leucocytes Scintigraphy. In the meanwhile, prophylactic antibiotic intravenously was administered on a daily basis and at the end of a haemodialityc session with antipyretic drugs (Tachipirine $1 \mathrm{gr}$ intravenously).

After bilateral nephrectomy surgery, the patient had slight temporary reduction of thermal curve and inflammation laboratory's indexes, which were previously constantly high as shown in Table 1.
Then, after 48 hours, the patient had an increasing fever $\left(39^{\circ} \mathrm{C}\right)$ and the laboratory tests showed anemia, decreasing in PCT, increasing in the leukocyte formula (with lymphocytopenia), slight decrease in the pool platelet compared to the conditions at the admittance, slight increase in Lactate dehydrogenase (LDH), slight increase in Protein C Reaction test $(P C R)$, non-specific increase in transaminases (GOT and GPT).

*Corresponding author: Ferrara G, Department of Nephrology and Dialysis, Fondazione IRCCS "Casa Sollievo della Sofferenza", San Giovanni Rotondo (FG), Italy

Received Date: April 20, 2020

Accepted: April 23, 2020

Published online: April 25, 2020

Citation: Ferrara G, Guida CC, De Cosmo S, et al. (2020) New Onset Viral COVID19 Infection in Two End Stage Renal Disease Patients on Extracorporeal Hemodialysis Treatment. Res Rev Infect Dis 3(1):53-57

Copyright: (c) 2020 Ferrara G, et al. This is an open-access article distributed under the terms of the Creative Commons Attribution License, which permits unrestricted use, distribution, and reproduction in any medium, provided the original author and source are credited. 
Citation: Ferrara G, Guida CC, De Cosmo S, et al. (2020) New Onset Viral COVID19 Infection in Two End Stage Renal Disease Patients on Extracorporeal Hemodialysis Treatment. Res Rev Infect Dis 3(1):53-57

Table 1: Laboratory's samples during recovery's time lapse.

\begin{tabular}{|c|c|c|c|c|c|c|c|c|c|}
\hline \multicolumn{3}{|c|}{ Patient 1} & \multirow{2}{*}{$\begin{array}{l}\text { Laboratory tests (recovery's time lapse) } \\
\text { TIME }\end{array}$} & \multicolumn{6}{|c|}{ Patient 2} \\
\hline T0 & T1 & T2 & & TO & T1 & T2 & T3 & T4 & T5 \\
\hline 3.090 & 3.130 & 2.460 & Red blood cells (n.v.: 4.070-6.010 mill/mcl) & 3.370 & 2.810 & 2.790 & 3.07 & 2.65 & 3.04 \\
\hline 9.3 & 8.9 & 7.2 & Haemoglobin (n.v.: 14-18 grams/deciliter) & 9.7 & 8.3 & 8.2 & 9.0 & 7.7 & 9.0 \\
\hline 12.6 & 24.460 & 18.670 & White blood cells (n.v.: 4.030-10.080 mill/mcl) & 10.350 & 5.47 & 4.52 & 5.29 & 3.83 & 3.71 \\
\hline 76.6 & 88.1 & 84.3 & Neutrophile (n.v. $40-80 \%$ ) & 82.9 & 81.5 & 78.6 & & & \\
\hline 12.3 & 6.1 & 8.3 & Lymphocite (n.v.: 10-45\%) & 10.3 & 7.8 & 14.5 & & & \\
\hline 416 & 511 & 305 & Platelets $(130-400 \times 1000 / \mathrm{mcl})$ & & & & 231 & 216 & 225 \\
\hline 2 & 1.1 & 1.7 & Uncoloured cells (<4\%) & 1.5 & 0.5 & 0.7 & & & \\
\hline neg & neg & neg & Blood culture & \multicolumn{3}{|c|}{ positive (Str. Mitis) } & & neg & \\
\hline 75 & 56 & 54 & Procalcitonine (<0 $0.5 \mathrm{mcg} /$ liters) & 5.44 & 2.03 & 1.46 & & 9.98 & \\
\hline \multirow[t]{2}{*}{11.2} & & 22.4 & PCR (C reactive protein $<0.30 \mathrm{mg} /$ deciliter) & 12.4 & 4.3 & & & & \\
\hline & & 0.57 & Endotoxinemia (0.40-0.60 U) & 0.75 & & 0.97 & & & \\
\hline 120 & 53 & 50 & VES (Erythrocite sedimentation rate $2-15 \mathrm{~mm}$ ) & 78 & & 74 & & & \\
\hline 37 & 445 & 46 & GOT (AST) (8-30 U/liters) & 15 & 20 & 18 & & & \\
\hline 36 & 132 & 50 & GPT (ALT) (13-57 U/liters) & 26 & 30 & 28 & & & \\
\hline 178 & 277 & 253 & LDH (Lactate dehydrogenase) & - & - & - & & & \\
\hline 60 & 213 & 143 & CPK (creatin phosphokinase) & - & - & - & & & \\
\hline
\end{tabular}

Due to the persistence of high fever $\left(>39^{\circ} \mathrm{C}\right)$ and due to COVID-19 pandemic period, nasal and oropharyngeal swab were carried out looking for COVID-19. The result was positive! Consequently he was placed in isolation and he was given personal protective equipment to ensure the personnel safety.

The following appearance of hemodynamic instability and worsening of respiratory acts, with the need for respiratory support with intubation, made necessary to transfer the patient into the intensive care unit in a sterile room. A temporary vascular access (right femoral vein) was positioned and the patient started a continuous hemodialysis treatment (CVVHDF) with high absorption filter (cytosorb) to remove the inflammatory mediators of the same acute phase in a treatment of three consecutive sessions [6-10].

The anticoagulant used in the "CRRT" hemodialysis session was seleparine (light weight molecular eparine) titrated on the dry weight and the time needed of the session.

All the health workers assigned to its control (nurses, medical doctors, others) were equipped with personal protective equipment (PPE or DPI). None of the hemodialized patient doing hemodialysis with him (before COVID-19 test) showed symptoms or fever. So where and mainly how did he get the infection? It could have occurred during the transfer from his own department to the radiology's one or during transfer to the hemodialysis' department.

Many cases and study report that the COVID-19 "flugge" and "droplet" can survive on many surfaces and areas from nine to fourteen hours before the viral load decreases by $50 \%$ of his contagiousness.

So the infection can happen in the transfer from a depart- ment to another or during and after the surgical session (even if the surgery room is ventilated with a negative pressure that avoids spreading, in addition to other measures to keep the room sterile for a surgery session).

At the admittance the patient one performed a C.T. - Total Body scan looking for any septic focus beyond the known data of infected cyst with consensual abscess. The result did not show a radiological imaging associable to lung viral infection by coronavirus (the "ground glass" and "crazy pavel" pattern as know).

He was examined also with a positron emission tomography imaging (SPECT) that showed increasing in leucocyte captation on suspected infected cists kidney's side.

The patient was dialyzed for nine days in the critical area in a continuous technique (continuous replacement therapy) and died on day ten.

The second case is a male Caucasian 68-years-old patient, affected by end stage renal disease and put on hemodialysis chronic treatment using an arterio-venous fistula. The fever was noted during the hemodialysis session in the clinic but also at home for about a week before the admittance to the hospital.

After performing a transthoracic cardiac ultrasound and a trans-esophageal study for the diagnosis of possible cardiac vegetations and structural alteration of the mitral valve (DUKE criteria), a computed vascular CT of the chest was performed that led to an endocarditis' diagnosis.

The blood culture sampled on day one showed the presence of "Streptococcus Mitis" and the patient started antibiotic intravenous therapy according to antibiogram in prevision of cardiac surgery. 
After about two weeks of antibiotic therapy and daily control of the thermal curve (periodic oscillations within $38^{\circ} \mathrm{C}$ ) he presented a new high fever appearance $\left(>39^{\circ} \mathrm{C}\right)$ associated with dry and irritating cough. These symptoms were not present at the admittance.

Suspecting a viral super-infection of COVID-19, in these critical pandemic period, a chest $\mathrm{X}$-ray and then an high resolution Chest-CT-scan (HRTC) was performed: It showed an highly specific and predictive imaging of viral infection by COVID-19 ("ground glass" radiological pattern in the lung bilaterally).

Laboratory tests showed anemization (despite of intravenous support with erythropoietin administration done at the end of any hemodialysis session so he performed at least one transfusion of concentrated red blood cells to cope with acute anemia), leukopenia with relative lymphocytosis, and decrease in PCR, PCT and VES, no alteration of transaminases.

At the same time, after consultation about infectious disease, the oropharyngeal and nasal swab was performed. Even in this case the result was positive.

Very difficult to know where he was infected. He was recovered in nephrology department and he was moved to the dialysis service (placed at the ground zero of the same building) and moved to the cardiac-surgery department for a hearth eco-scanning.

In all such cases he was transferred wearing a mask since the OMS declared the world-wide pandemic $\left(11^{\text {th }}\right.$, March 2020).

So he was quickly placed into isolation and he continued replacement hemodialysis therapy wearing individual protection devices inside a dedicated dialysis room. The other outpatients had no symptoms or fever and continued their own hemodialysis weekly sessions wearing mask just entering the clinic and washing accurately their hands and the fistula in the arm with alcoholic sterile solution before starting hemodialysis session.
In this patient it was observed a reduction of the thermal curve in the first phase of admission (after endocarditis' diagnosis and antibiotics therapy). Subsequently a stable body high temperature was measured $\left(39^{\circ} \mathrm{C}-40{ }^{\circ} \mathrm{C}\right)$ and antipyretics drugs treatment was started at high doses intravenously (Tachipirine 1 gram per I.V. up to four times per day and/or acetylsalicylic acid at the dose of $250 \mathrm{mg}$ per I.V. up to one gram per day).

A nowadays patient is still alive and does hemodialysis on three day/week rhythm in a dedicated COVID department room.

\section{Discussion}

The epidemic of coronavirus disease 19 (COVID-19) has spread rapidly around the globe with considerable morbidity and mortality. So the co-infection can be very frequent in the general population, particularly in patients with older age and several comorbidities.

Previous studies assume that COVID-19 infected patients with chronic comorbidities can more easily have severe complications [11,12]. Patients with end-stage renal disease who are dialysis dependent can be at great risk of COVID-19 infection due to suppression of the immune system. It has been suggest that T-cell immunity plays an important role and the uremia status is associated with extensive impairment of lymphocyte and granulocyte function. An abnormal immune system can alter their response to Sars-CoV infection [13].

In this report we describe two COVID-19-infected hemodialysis patients who are referred in our hospital for different pathologies. During hospitalization both patients presented fever and the increase of inflammation markers. CT of the chest was done (Figure 1 and Figure 2) and COVID-19 testing was performed. The result of which were positive for COVID-19 infection.

The two patients were isolated and moved into dedicated dialysis room. Both of them continued extracorporeal replacement hemodialysis therapy, one in a continuous mode
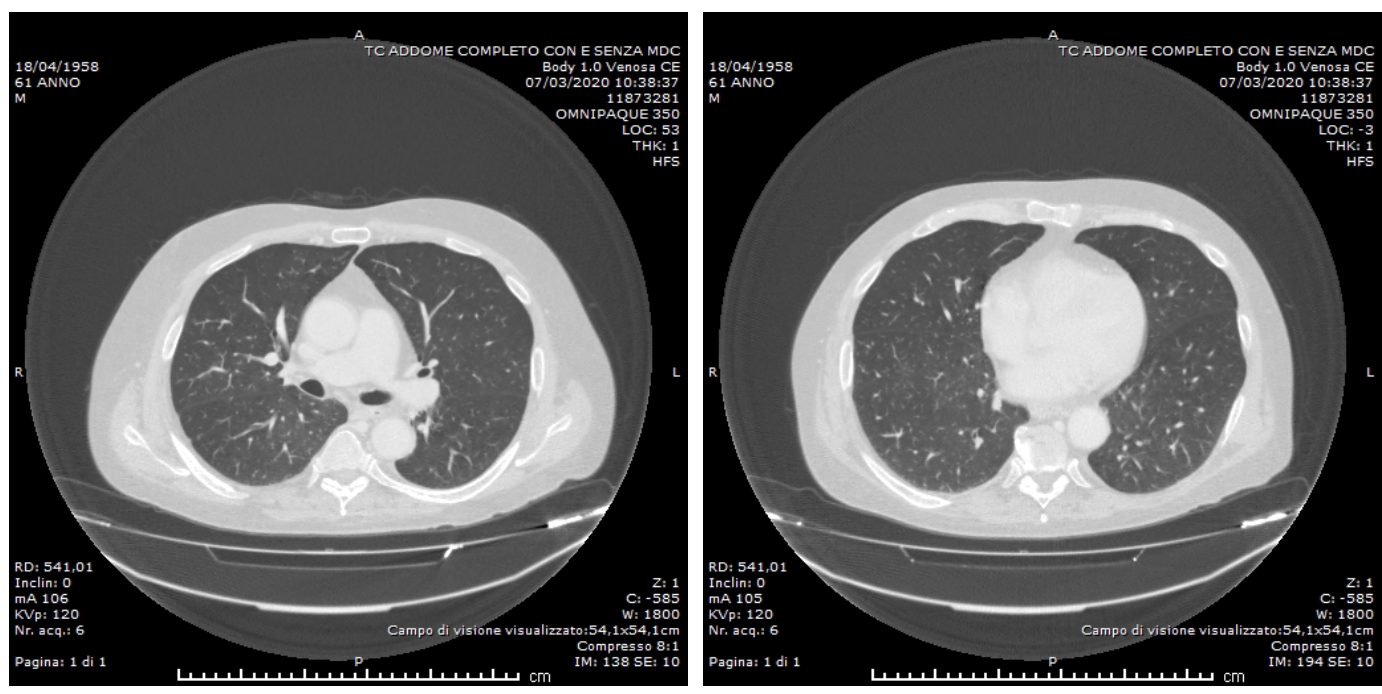

Figure 1: Computed tomography lung scan showed standard radiological image at admittance. 

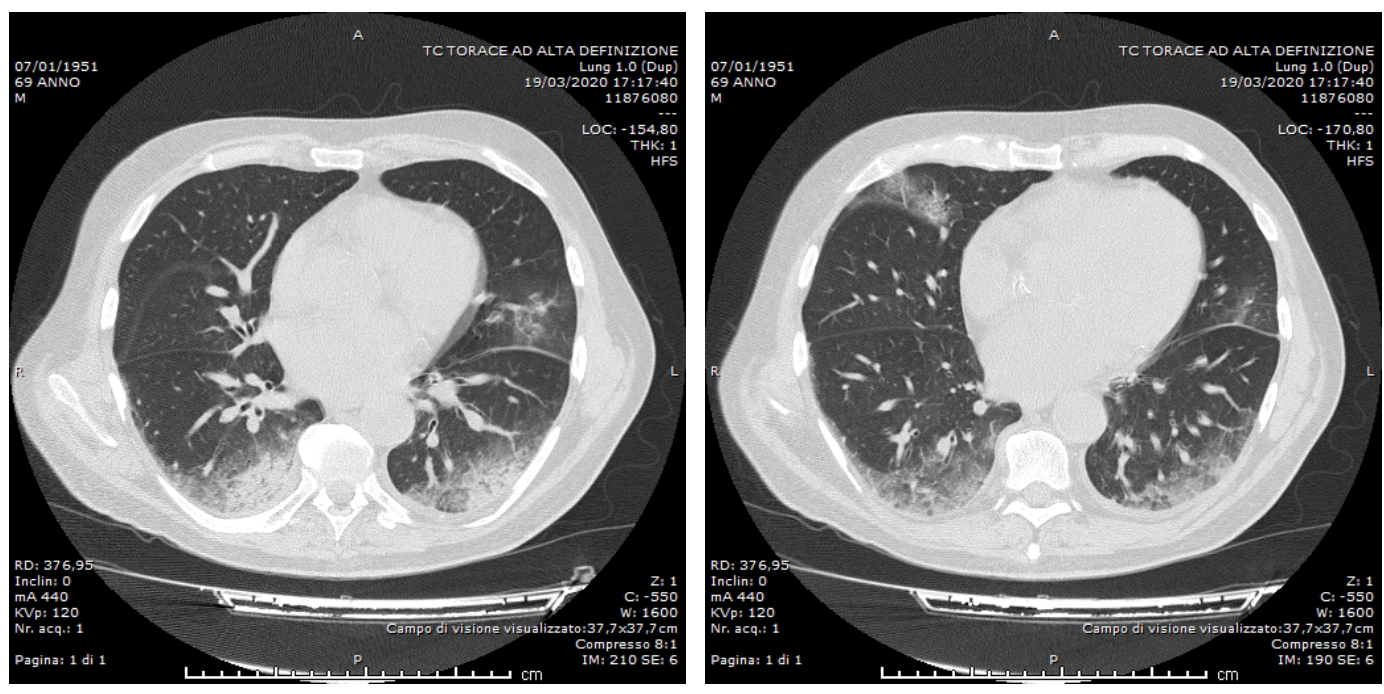

Figure 2: Thoracic panel coherent with lung infection by COVID-19 during hospitalization.

in the intensive care unit and the other one in a dedicated dialysis chamber. The goal was to isolate these cases to minimize the risk of local clustered transmission. Moreover several measures had been taken: The use of masks for all health workers and patients and drivers of organized transport were instructed not to transfer patients, unless masks were worn.

We assume COVID-19 infection could be happen during transfer from their own department to others or from any asymptomatic carriers (nurse, doctors, health workers) that had contact with them.

This case report highlights the importance of considering COVID-19 infection in a variety of clinical presentation that may not initially include typical respiratory symptom to prevent ongoing exposure of potentially affected individuals to the general population. It is also important the need for rapidly available testing for all patients who present to healthcare setting [14].

In some high-risk populations, such as dialysis patients, where several individuals are treated at the same time in a limited space and overcrowded areas, our objective must be to ensure protection to patients, the healthcare team and the dialysis ward [15].

So you have to periodically check your own patients but also colleagues and workers to avoid spreading of infection and keep them in quarantine or give them adequate protection (washing the hand, do not touch mouth, eyes or nose with hands, keep on masks).

There is no specific antiviral treatment recommended for COVID-19, and no vaccine is currently available on humans [16]. The treatment is symptomatic, and oxygen therapy represents the major treatment intervention for patients with severe infection. In Italy, a great investigation led by the "Istituto Nazionale Tumori - Fondazione Pascale di Napoli" is focused on the use of Tolicizumab. It is a humanized IgG1 monoclonal antibody, directed against the IL- 6 receptor and commonly used in the treatment of rheumatoid arthritis [3].
COVID-19 is a challenge to our health systems because of its novelty, rapidity of spread and mortality. This pandemic has required and continues to require broad attention of public health official to implement social distancing to mitigate the rapid spread of this highly contagious and potentially life-threatening novel virus, particularly for the people at risk of developing complications.

\section{References}

1. He F, Deng Y, Li W (2020) Coronavirus disease 2019: What we know? J Med Virol.

2. Sun $P$, Lu X, Xu C, et al. (2020) Understanding of COVID-19 based on current evidence. J Med Virol.

3. Cascella M, Rajnik M, Cuomo A, et al. (2020) Features, evaluation and treatment coronavirus (COVID-19). StatPearls Treasure Island.

4. Young BE, Ong SWX, Kalimuddin S, et al. (2020) Epidemiologic features and clinical course of patients infected with SARS-CoV-2 in Singapore. JAMA.

5. Report of the WHO-China Joint Mission on Coronavirus disease 2019 (COVID-19).

6. Ronco C, Navalesi P, Vincent JL (2020) Coronavirus epidemic: Preparing for extracorporeal organ support in intensive care. Lancet Respir Med 8: 240-241.

7. Bottari G, Merli P, Guzzo I, et al. (2020) Multimodal therapeutic approach of cytokine release syndrome developing in a child given chimeric antigen receptor-modified T cell infusion. Crit Care Explor 2: e0071.

8. Napp LC, Ziegeler S, Kindgen-Milles D (2019) Rationale of hemoadsorption during extracorporeal membrane oxygenation support. Blood Purif 48: 203-214.

9. Brouwer WP, Duran S, Kuijper M, et al. (2019) Hemoadsorption with CytoSorb shows a decreased observed versus expected 28day all-cause mortality in ICU patients with septic shock: A propensity-score- weighted retrospective study. Crit Care 23: 317.

10. Friesecke S, Stecher SS, Gross S, et al. (2017) Extracorporeal cytokine elimination as rescue therapy in refractory septic shock: $A$ prospective single-center study. J Artif Organs 20: 252-259. 
Citation: Ferrara G, Guida CC, De Cosmo S, et al. (2020) New Onset Viral COVID19 Infection in Two End Stage Renal Disease Patients on Extracorporeal Hemodialysis Treatment. Res Rev Infect Dis 3(1):53-57

11. Adalja AA, Toner E, Inglesby TV (2020) Priorities for the US health community responding to COVID-19. JAMA.

12. Huang C, Wang Y, Li X, et al. (2020) Clinical features of patients infected with 2019 novel Coronavirus in Wuhan, China. Lancet 395: 497-506.

13. Zhou J, Li C, Zhao G, et al. (2017) Human intestinal tract serve as an alternative infection route for Middle East respiratory syndrome coronavirus. SCI ADV 3: eaao4966.

14. Ferrey AJ, Choi G, Hanna RM, et al. (2020) A case of novel coro- navirus disease 19 in a chronic hemodialysis patient presenting with gastroenteritis and developing severe pulmonary disease. Am J Nephrol 28: 1-6.

15. Rombolà G, Heidempergher M, Pedrini L, et al. (2020) Correction to: Practical indications for the prevention and management of SARS-CoV-2 in ambulatory dialysis patients: Lessons from the first phase of the epidemics in Lombardy. J Nephrol 30: 1.

16. De Wit E, Feldmann F, Cronin J, et al. (2020) Prophylactic and therapeutic remdesivir (GS-5734) treatment in the rhesus macaque model of MERS-CoV infection. PNAS 117: 6771-6776. 